\title{
JAK/STAT pathway in pathology of rheumatoid arthritis (Review)
}

\author{
DANA ALEXANDRA CIOBANU ${ }^{1 *}$, IOAN SABIN POENARIU ${ }^{2 *}$, LAURA-IOANA CRINGGUȘ ${ }^{1}$, \\ FLORENTIN ANANU VREJU ${ }^{1}$, ADINA TURCU-STIOLICA ${ }^{3}$, ANDREI ADRIAN TICA ${ }^{4}$, \\ VLAD PADUREANU ${ }^{5}$, ROXANA MIHAELA DUMITRASCU ${ }^{1}$, SIMONA BANICIOIU-COVEI ${ }^{1}$, \\ STEFAN CRISTIAN DINESCU ${ }^{1}$, LIDIA BOLDEANU ${ }^{6 *}$, ISABELA SILOȘI ${ }^{2}$, \\ ANCA MARILENA UNGUREANU ${ }^{6}$, MIHAIL VIRGIL BOLDEANU ${ }^{2,7}$, \\ EUGEN OSIAC $^{8}$ and ANDREEA LILI BARBULESCU ${ }^{4}$ \\ Departments of ${ }^{1}$ Rheumatology, ${ }^{2}$ Immunology, ${ }^{3}$ Pharmacoeconomics, ${ }^{4}$ Pharmacology, \\ ${ }^{5}$ Medical Semiology and ${ }^{6}$ Microbiology, University of Medicine and Pharmacy of Craiova, 200349 Craiova; \\ ${ }^{7}$ Medico Science SRL-Stem Cell Bank Unit, 200690 Craiova; ${ }^{8}$ Department of Biophysics, \\ University of Medicine and Pharmacy of Craiova, 200349 Craiova, Romania
}

Received June 3, 2020; Accepted July 3, 2020

DOI: $10.3892 /$ etm.2020.8982

\begin{abstract}
Rheumatoid arthritis (RA) is classified as an inflammatory, chronic autoimmune and disabling disease based on the intricate interplay between environmental and genetic factors. With a prevalence ranging from 0.3 to $1 \%$, RA is the most prevalent inflammatory joint disease observed in adults. Disruption of immune tolerance becomes evident when abnormal stimulation of the innate and adaptive immune system occurs. This cascade of events causes persistent joint inflammation, proliferative synovitis and, ultimately, damage of the underlying cartilage as well as the subchondral bone, leading to permanent joint destruction, deformity and subsequent loss of function. With cytokines being the key to a multitude of biological processes, including inflammation, hematopoiesis and overall immune response, one must inevitably look at the main pathways through which a significant number of those molecules exert their function. Janus kinase/signal transducers and activators of transcription (JAK/STATs) represent one such pathway and, recently, JAK inhibitors (JAKinibs) have shown promise in the treatment of several inflammatory diseases, including RA. This
\end{abstract}

Correspondence to: Professor Mihail Virgil Boldeanu, Department of Immunology, University of Medicine and Pharmacy of Craiova, 2-4 Petru Rares Street, Dolj, 200349 Craiova, Romania

E-mail: boldeanumihailvirgil@yahoo.com

Professor Florentin Ananu Vreju, Department of Rheumatology, University of Medicine and Pharmacy of Craiova, 2-4 Petru Rares Street, Dolj, 200349 Craiova, Romania

E-mail: florin_vreju@yahoo.com

${ }^{*}$ Contributed equally

Key words: rheumatoid arthritis, JAK/STAT pathway, interleukins, cytokine signaling, JAK inhibitors, pharmacotherapy narrative review focuses on the intricate signaling pathways involved as well as on the clinical aspects and safety profiles of JAKinibs approved for the treatment of RA.

\section{Contents}

1. Introduction

2. Pathogenesis and cytokine signaling in rheumatoid arthritis

3. Janus family of kinases and JAK/STAT pathway

4. Rheumatoid arthritis-pharmacotherapy

5. Conclusions

\section{Introduction}

Autoimmune diseases occur as a result of the imbalance between proinflammatory and anti-inflammatory cytokines, secondary to a breakdown of immunologic tolerance of autoreactive immune cells. A good comprehension of the pathogeny, an early diagnosis, together with a careful holistic evaluation (1-3) and an optimal treatment approach might avoid future complications and permit the best management in autoimmune diseases.

Rheumatoid arthritis (RA), one of the most frequent autoimmune disease, is a systemic, polyarticular, chronic, progressive inflammatory musculoskeletal disorder of the synovial joints. In addition to joint inflammation, considerable tissue damage associated with RA can occur in the heart, as well as in the lungs, skin, eyes, kidneys, and blood vessels (4).

Abnormal proliferation results in an aberrant presence of activated T lymphocytes, B lymphocytes, mast cells, neutrophils, macrophages, and accessory antigen presenting cells [dendritic cells (DCs)], as well as synovial tissue fibroblasts [fibroblast-like synoviocytes (FLSs)], which are the cardinal cellular signs of the rheumatoid disease process (5). 
Significantly increased levels of pro-inflammatory cytokines, i.e. tumor necrosis factor- $\alpha$ (TNF- $\alpha$ ), interleukin (IL)-1 $\beta$, IL-6, IL-8, IL-12/IL-23, IL-17, IL-18, IL-32 and interferon- $\gamma($ IFN- $\gamma)$ produced by different cells, together with growth factors, such as fibroblast growth factor-2 (FGF-2) and the vascular endothelial growth factor (VEGF), the latter produced mainly by synovial-like fibroblasts and macrophages, has been shown to be crucial for RA to progress clinically, where destruction of articular cartilage and erosions of the subchondral bone are the main events that result in synovial joint failure (6).

Several signal transduction pathways have been implicated in RA progression. For example, although it is observed that IL-1 $\beta$ predominantly activates the stress-activated/mitogen-activated protein kinases (SAPK/MAPK) and IL- 6 and IFN- $\gamma$ predominantly activate Janus kinase/signal transducers and activators of transcription (JAK/STAT), convincing evidence indicates activation of MAPK signaling by IL-6, IL-22 and IFN- $\gamma(7,8)$. It was reported that TNF- $\alpha$ activated mainly SAPK/MAPK, but also the JAK/STAT pathway, as shown in the results obtained, according to which the recombinant (rh)-TNF- $\alpha$, causes, in vitro, the phosphorylation of STAT3 protein (p-STAT3) at the level of human chondrocytes without modifying the STAT3 content (9). Of note, the activation of JAKs by IL- 6 was also related to the activation of the SAPK/MAPK pathway and PI3K/Akt/mTOR signaling through 'cross talk', whereby the PI3K/Akt/mTOR pathway, in particular, has been associated with aberrant survival of immune cells in RA (10).

The crucial role played by the activation of the JAK/STAT pathway in RA was further confirmed after the United States Food and Drug Administration (FDA) approval of the selective small molecule inhibitor (SMI) of JAK3, tofacitinib, for medical therapy in RA. Indeed, successful incorporation of tofacitinib into the fold of RA therapies, has led to further development of JAK1-selective, JAK2-selective, tyrosine kinase 2-selective (TYK2) and pan-JAK SMI $(7,11)$.

\section{Pathogenesis and cytokine signaling in rheumatoid arthritis}

Normal balance between inflammation and homeostasis is maintained through cellular adaptation and the use of intracellular signaling mechanisms, which consists of a series of interactions between intracellular proteins. Transmission of information is achieved through chemical signals coming from outside the cell, relayed along the pathway towards the cell nucleus, ultimately leading to gene activation through transcription $(12,13)$.

These molecules play a vital role in the pathogenesis of several autoimmune diseases such as inflammatory bowel disease (IBD), RA, spondyloarthritis group (SpA), several skin conditions and others. Clear insights have been gained over the last three decades which point to a range of diverse cytokines as being the key driving force behind several autoimmune diseases, including RA. The JAK-STAT pathway facilitates the signal transduction of various cytokines and molecules $(4,14-16)$.

Interleukins. TNF- $\alpha$, IL-1 and IL-17 are essential in immune-mediated diseases and host defense, however, they do not signal using JAKs and STATs. Specific cytokines, which are dependent on the JAK/STAT pathway, are able to induce TNF- $\alpha$, IL-1 and IL-17. IL- 6 stimulates the production of both IL-1 and TNF- $\alpha$, while further inducing the differentiation of the T helper type 17 (Th17 cells) subset, which are themselves significant producers of IL-17. Moreover, TNF- $\alpha$ acts synergistically with IL-17 in order to increase the level of matrix metalloproteinases (MMPs) and activate FLSs and chondrocytes (4,17-19).

The IL-6 family is also known as gp130 cytokines due to the fact that representatives of this family use, as a signal transducer, glycoprotein 130. It consist of IL-6, IL-11, IL-27 and IL-31, but also other factors such as oncostatin M (OSM), cardiotrophin-1 (CT-1), leukemia inhibitory factor (LIF), neuropoietin (NP), cardiotrophin-like cytokine (CLC) and ciliary neurotrophic factor (CNTF). Gp130 cytokines are implicated in the modulation of several processes such as neuronal survival, cardiovascular activity, immune system response and inflammation $(15,20)$.

Specifically, IL-11 participates in bone remodeling and hematopoiesis. The T helper type- 2 cells (Th2-cells) and mast cells produce IL-31, which regulates myeloid development and skin functions. IL-27, on the other hand, decreases inflammation by antagonizing the production of IL-17 $(1,21)$. One of the cytokines belonging to the IL- 6 family, oncostatin, exhibits anti-inflammatory properties in collagen-induced arthritis by inhibiting Th17-cells, through its effect on STAT3, STAT5 and the proteins of the suppressor of cytokine signaling 3 (SOCS3). The SOCS regulate JAK/STAT signaling $(15,20)$.

IL-6. IL-6 is the prototypical, pleiotropic, pro-inflammatory cytokine, which, as with other related cytokines, uses the gp130 receptor subunit for signaling purposes. It has a multitude of biological functions, from acute inflammatory and immune responses to being implicated in nervous system development, as well as hematopoietic development $(4,22)$. Elevated levels of IL- 6 are seen in a number of inflammatory diseases such as RA. Phosphorylation of the STAT1 and STAT3 proteins, mediated by JAK1 and JAK2, is induced by IL-6 $(23,24)$.

The gp130 receptor is known as a receptor subunit and as a signal transducer pertaining to the IL- 6 family of cytokines. It is involved in the JAK/STAT signaling pathway as an activator of STAT3, which has an essential role in cellular differentiation and growth, as well in the process of osteoclastogenesis. Therefore, modulation of this signaling pathway (IL-6/gp130/STAT3) is regarded as a compelling therapeutic avenue for RA treatment (23).

The Thelper type 17 cells (Th17 cells). The Th17-cells play a crucial role as mediators of autoimmunity, with IL-2 being a decisive inhibitory factor for their differentiation. Through the STAT5 mechanism, IL-2 suppresses the expansion of Th17 clones, which are antigen-specific (25-27). In RA, patients present with an altered profile when compared with healthy donor cells, a fact observed with a higher expression level of the IL-1 receptor type 1 (IL-1R1), IL-23 receptor (IL-23R), JAK2, C-C motif chemokine ligand 20 (CCL20) and the colony-stimulating factor 2 (CSF2) [granulocyte-macrophage colony-stimulating factor 2 (GM-CSF2)]. JAK inhibitors (JAKinibs) may prove to be useful in lowering Th17 
cell responses, as various pathways which compel the differentiation of Th17-cells, also implicate the cytokine-bound JAK-STAT signaling pathway (28).

IFNs. IFNs represent a group of cytokines exhibiting antimicrobial, antiviral, antitumor and antiproliferative effects, acting as modulators for both the innate and the adaptive immune system. They are classified into different types and subtypes, depending on sequencing homology, mapping of peptides and interactions with the IFN receptor subunits. IFN- $\alpha$, IFN- $\beta$ and IFN- $\lambda$ have a strong antiviral effect through modulation of cell activity and inhibition of viral propagation. However, type II IFN class, specifically IFN- $\gamma$, acts mainly as a macrophage-activator, despite exhibiting antiviral activity $(12,29)$. JAK1 and JAK 2 are utilized by the IFN $-\gamma$ receptor in order to phosphorylate STAT1 (9).

Besides the receptors for the type I $(\alpha, \beta$ and $\omega)$ and type II $(\gamma)$ interferons (IFNs), a receptor complex, which binds type III IFN, complex formed by the IL-28R $\alpha / \mathrm{IFN}-\lambda$ R1 ligand-binding subunit and the IL-10R $\beta$ accessory chain, have also been identified. The type III IFN receptor mediates IL-10, IL-22 and IL-26 signaling. Moreover, it has been determined that TYK2 and JAK1 are associated with IL-10R2. There is now evidence to suggest that apart from the standard JAK-STAT signaling pathway, IFN stimulation is also associated with MAPK pathway, phosphatidyl-inositol 3-kinase (PI3K) pathway, the Crk-like protein (CrkL) and Ras related protein 1 (RAP1) pathway (30).

FLSs. FLSs produce cytokines capable of generating and maintaining autoimmune inflammation, as well as proteases which advance the destruction of cartilage (22). Located in the hyperplastic synovium, RA-derived FLSs cause considerable cartilage invasion (31). As such, synovial joint failure occurs due to loss of articular cartilage, along with the subchondral bone erosion (32). A study revealed that after a $24 \mathrm{~h}$ treatment using chlorogenic acid (CGA), the expression levels of JAK1, gp130, and p-STAT3, induced by IL-6, were greatly suppressed in FLS cells (22).

Recently, several studies have highlighted the importance of cell-to-cell contact when it comes to crosstalk and interaction between various cell types. Mori et al established that this particular differentiation, along with the commitment into Th1 cells is inducible through the cell-to-cell contact of naive T lymphocytes and FLS using adhesion molecules (33). Joint FLS in RA possess unique transcriptomes, epigenetic markers, as well as a STAT3 activation sequence, pertaining to the IL-6 pathway. The aforementioned factors modulate the clinical response seen with therapies involving JAK inhibitors (34).

\section{Janus family of kinases and JAK/STAT pathway}

JAKs are a group consisting of 4 members, namely JAK1, JAK2, JAK3, as well as tyrosine kinase 2 (TYK2) and are tyrosine kinase non-receptor proteins. Their name is based on the two-faced Roman duality God 'Janus', because the JAKs possess two near-identical phosphate-transferring domains. These cytoplasmic tyrosine kinases are capable of phosphorylating tyrosine residues either through transphosphorylation or autophosphorylation. Despite having been initially classified as 'just another kinase', JAKs represent the principal initiators of the later named JAK/STAT pathway (14).

The IFNs and the JAK/STAT pathway are integrated into different signal transduction pathways by communicating with innate pattern recognition receptors (PRRs). PRRs include C-type lectin receptors (CLRs), retinoic-acid inducible gene I (RIG)-I-like receptors (RLRs), Toll-like receptors (TLRs) as well as nucleotide-binding oligomerization domain (NOD)-like receptor (NLRs) (29).

Relating to TYK2, a loss of function mutation is known to cause primary immunodeficiency. STAT2 deficiency causes an increased susceptibility to viral mutations and STAT4 polymorphisms are associated with both RA and systemic lupus erythematosus (SLE) (35).

The JAK/STAT signaling pathway not only regulates cytokine signaling but is also implicated in the production of anti-inflammatory cytokines, thereby having a key role in the signaling related to neuropoietic cytokines $(36,37)$. This pathway provides a similar role in the pathogenesis of RA, whereby JAK molecules can activate specific immune cells and induce the expression of pro-inflammatory cytokines, with regulation being achieved through JAK1 and JAK3 through cytokine binding (38).

Following the receptor binding during a cytokine response, the first intracellular signaling molecules that become activated are tyrosine kinases, several being implicated in the inflammatory response. As such, in recent years, focus has shifted to these tyrosine kinases for their potential as a therapeutic target in RA (39).

STATs represent a prominent class of molecules which can transmit signals from cytokine receptors (type I and II) to the nucleus and prior to activation, they reside in the cytoplasm as inactive proteins. Upon cytokine activation, they bind active, phosphorylated receptors (IL-6 to the IL-6R $\alpha / g p 130$ ), becoming subsequently phosphorylated by JAKs. Then, STATs translocate to the nucleus and bind the deoxyribonucleic acid (DNA), thus activating the transcription of several specific target genes $(4,32)$. Seven STATS are currently known: STAT1, STAT2, STAT3, STAT4, STAT5A, STAT5B, and STAT6. In a similar fashion to JAKs, STATs handle the transmission of signals for various cytokine receptors (40).

In particular, STAT3 is regarded as a pathogenic factor in RA due to its ability to promote angiogenesis, inhibit fibroblasts apoptosis and promote the expression of certain matrix metalloproteinases such as MMP-2 and MMP-9 (41).

\section{Rheumatoid arthritis-pharmacotherapy}

To limit impairment, current RA treatment guidelines focus on a combination of drugs, education on managing symptoms, implementing an exercise routine with sufficient resting periods and lastly, surgical intervention, when required. Medication used in the treatment of RA includes: synthetic disease-modifying anti-rheumatic drugs (DMARDs), biologic agents such as TNF- $\alpha$ inhibitors, interleukin inhibitors (IL-6 inhibitor), B cell-depleting agents, targeted synthetic disease-modifying anti-rheumatic drugs (tDMARDs) such as Janus kinase inhibitors (JAKinibs), along with symptomatic, considered to be fast-acting drugs i.e. nonsteroidal anti-inflammatory drugs (NSAIDs) and glucocorticoids $(38,42,43)$. 
Between the DMARDs, methotrexate (MTX), the initial, golden standard drug, is an analog to folic acid that competitively inhibits the binding of dihydrofolic acid (FH2) to the enzyme that is responsible for converting $\mathrm{FH} 2$ to folinic acid (FH4). The lack of FH4 leads to impairment of the metabolism of purine and pyrimidine, and to inhibition of amino acids and polyamine synthesis (44-46). The DMARD family includes, besides MTX, hydroxychloroquine, sulfasalazine and leflunomide, a drug which inhibits the synthesis of ribonucleotide uridine monophosphate pyrimidine.

The biologic DMARDs are bigger molecules, genetically engineered versions of naturally-occurring substances, or novel compounds which represent a more 'direct, defined and targeted' therapeutic approach. The biologics class include tumor necrosis factor inhibitors, such as monoclonal antibodies (infliximab, adalimumab, certolizumab, golimumab) and fusion proteins (etanercept), IL-6 inhibitors (tocilizumab), B-cell depleting drugs targeting CD20 marker (rituximab), or blockers of the CD28/B7 pathway by cytotoxic T-lymphocyte-associated protein 4-Ig [CTLA4-Ig (abatacept)] and have shown to be very effective in patients with rheumatoid arthritis.

JAKinibs. Several cytokines involved in RA pathogenesis, such as IL-6, bind to specific receptors that are in a direct relation with JAK-STAT pathway and signal transduction. Binding of different cytokines to specific receptor leads to activation of specific JAK pathways. Thus, IL-2, IL-7, IL-9 have associated JAK1, JAK3 and STAT3 and STAT5, while IL-6 and IL-11 activates JAK1, JAK2, TYK2 with STAT1, STAT3 pathway. Different JAK/STAT activation is linked to specific effect, in inflammation, erythropoiesis, leukocyte maturation and in the body defense against infections, some of them incompatible with life.

Targeting selectively different types of JAK with low molecular drugs, available as oral preparations, allows modulation of the inflammatory and immune responses.

There are currently two JAKinibs approved for RA treatment, tofacitinib (selective for JAK1 and JAK3) and baricitinib (selective for JAK1 and JAK2), as other agents are undergoing clinical studies: upadacitinib and filgotinib (selective for JAK1), peficitinib (selective for JAK1 and JAK2), decernotinib (JAK3 selective) (47-51).

The selectivity for JAK isoform is relative and the agents already approved are effective consecutive to JAK1 inhibition, although it implies a possible risk of herpes zoster reactivation. JAK1 interferes with signal transduction for several cytokines, IL- 6 being proved to be a target in RA management. Inhibiting JAK2 might be associated with some of the side effects, including platelet aggregation and the possible role for thrombotic risk, or inhibiting erythropoietic process $(48,51)$.

Tofacitinib. It is a targeted small molecule, which interferes with JAK-dependent cytokine signaling and modulating the inflammatory and immune responses, along with cytokine production, with consequent effect on lymphocytes, natural killer (NK) and B cells $(47-49,52,54)$. The first report of tofacitinib was published by Borie $e t a l$, as a JAKinib was used to prevent transplant rejection, according to the results of several studies, which validated its efficacy; tofacitinib significantly improved allograft survival in several primate studies (54).
It was the first JAKinib approved in 2012 for moderate to severe active RA, in patients with inadequate response to Methotrexate. Its main activity is as JAK1/JAK3 inhibitor, with a potential effect on JAK2 and a neglectable one on TYK2 (55).

Tofacitinib, in doses of 5 and $10 \mathrm{mg}$ twice a day, was the subject of several safety and efficiency studies, with common side effects represented by upper respiratory tract infections, cephalalgia, diarrhea, reactivation of herpes zoster or malignancies (52-58). Regarding laboratory parameters, its administration can be associated to changes in lipids profile, increased creatinine or hepatic enzymes; also reported was a decrease of neutrophil count, within the first 3 months of treatment, along with changes of circulating lymphocyte subsets (51).

Baricitinib, is a selective JAK1/JAK2 inhibitor, with effects on several pro-inflammatory cytokines, i.e. IL-6, IL-12, IL-23 and IFN- $\gamma$. It is administered in a dosage of $4 \mathrm{mg}$ daily, with or without methotrexate, with proven efficacy on structural joint damage (59); the most common side effects are similar to the ones of tofacitinib, and include respiratory infections, altered lipid profile, decreased hemoglobin and neutrophils, increased hepatic enzymes or creatinine $(60,61)$. Due to less affinity for JAK3, it may be associated to fewer immunosuppressive effects $(52,60,61)$. Its administration can be associated to infectious events, increased cholesterol (total, low-density lipoprotein, high-density lipoprotein), triglycerides, creatinine, or hepatic transaminases, as well as haemoglobin changes, consequent to erythropoiesis inhibition.

Filgotinib is another selective JAK1/JAK2 inhibitor, with a 30-fold selectivity towards JAK1, that demonstrated efficiency regarding disease activity, assessed using American College of Rheumatology (ACR) 20/50, Disease Activity Score-C-Reactive Protein (DAS28-CRP) or Simple Disease Activity Index (SDAI). The data observed across trials showed that the safety profile was encouraging, similar to other selective JAK1 inhibitors and the most commonly reported side effects were represented by upper respiratory tract infections (62).

Upadacitinib is an oral compound, with selectivity for JAK1, that showed a favorable efficacy, safety and tolerability in the studies conducted so far; consequent to its administration, the radiographic progression in RA patients was reduced, along with important improvement of life quality and physical function. The most frequently seen side effects were represented by cephalalgia, infections (respiratory or urinary) or nausea (62).

Peficitinib is a new oral compound, moderately selective for JAK3, that showed a favorable safety profile. Early clinical studies reported promising results and no significant major adverse effects (63).

Ruxolitinib, initially used for the treatment of polycythemia vera and primary myelofibrosis, is a JAK1 and JAK2 inhibitor with encouraging effects in RA patients $(64,65)$.

Decernotinib, an oral selective JAK3 inhibitor, with a 5-fold selectivity compared with JAK1, JAK2 and TYK2, is currently under study for the treatment or RA. Regarding safety, the side effects were reported directly related to dose and were mostly represented by nausea, cephalalgia, increased cholesterol levels, aminotransferases or respiratory infections $(66,67)$.

The challenge of managing RA patients using JAK inhibitors should carefully balance the risks and benefits of JAK2, JAK3 and TYK2 suppression, and future studies are required 
in order to choose the proper agent that can offer effectiveness and fewer side effects.

\section{Conclusions}

The efforts for better knowledge of RA pathogenesis have shown results progressively, with the current possibility of a personalized therapeutic approach, in order to obtain a better outcome, prevent future complications and improve the quality of life. Any small step in understanding the disease pathogenesis, might be beneficial as ongoing research will find different treatment targets and will surely establish the proper selectivity, optimized dosing, and why not patient selection, in order to minimize undesirable adverse effects and maximize the outcome.

\section{Acknowledgements}

Not applicable.

\section{Funding}

No funding was received.

\section{Availability of data and materials}

Not applicable.

\section{Authors' contributions}

DAC, ISP, LIC, FAV, ATS, ADT, VP, RMD, SBC, SCD, LB, IS, AMU, MVB, EO and ALB contributed to the acquisition, analysis and systematization of the data, wrote the manuscript and critically revised it for important intellectual content. All authors read and approved the final version of the manuscript.

\section{Ethics approval and consent to participate}

Not applicable.

\section{Patients consent for publication}

Not applicable.

\section{Competing interests}

The authors declare that they have no competing interests.

\section{References}

1. Smith DA and Germolec DR: Introduction to immunology and autoimmunity. Environ Health Perspect 107 (Suppl 5): 661-665, 1999.

2. Mustafa ER, Firulescu SC, Parvanescu C, Chisalau B, Tartea G, Efrem I, Barbulescu A, Dinescu S, Ciurea P, Radu L, et al: Triple valve infective endocarditis - a late diagnosis. J Mind Med Sci 5: 141-144, 2018.

3. Firulescu SC, Tudorașcu DR, Pârvănescu $C D$, Chisălău AB, Bastian AE, Efrem IC, Bărbulescu AL, Forţofoiu MC, Criveanu C, Ionescu P, et al: The role of skin and muscle biopsy in the diagnosis of main connective tissue diseases. Rom J Morphol Embryol 59: 55-64, 2018.

4. Schwartz DM, Bonelli M, Gadina M and O'Shea JJ: Type I/II cytokines, JAKs, and new strategies for treating autoimmune diseases. Nat Rev Rheumatol 12: 25-36, 2016.
5. Schwartz DM, Kanno Y, Villarino A, Ward M, Gadina M and O'Shea JJ: JAK inhibition as a therapeutic strategy for immune and inflammatory diseases. Nat Rev Drug Discov 17: 78, 2017.

6. Fleischmann R, Mysler E, Hall S, Kivitz AJ, Moots RJ, Luo Z, DeMasi R, Soma K, Zhang R, Takiya L; and ORAL Strategy investigators: Efficacy and safety of tofacitinib monotherapy, tofacitinib with methotrexate, and adalimumab with methotrexate in patients with rheumatoid arthritis (ORAL Strategy): a phase $3 \mathrm{~b} / 4$, double-blind, head-to-head, randomised controlled trial. Lancet 390: 457-468, 2017.

7. Fleischmann R, Kremer J, Cush J, Schulze-Koops H, Connell CA, Bradley JD, Gruben D, Wallenstein GV, Zwillich SH and Kanik KS; ORAL Solo Investigators: Placebo-controlled trial of tofacitinib monotherapy in rheumatoid arthritis. N Engl J Med 367: 495-507, 2012.

8. Vieira MC, Zwillich SH, Jansen JP, Smiechowski B, Spurden D and Wallenstein GV: Tofacitinib versus biologic treatments in patients with active rheumatoid arthritis who have had an inadequate response to tumor necrosis factor inhibitors: Results from a network meta-analysis. Clin Ther 38: 2628-2641.e5, 2016.

9. Eisenstein EM and Berkun Y: Diagnosis and classification of juvenile idiopathic arthritis. J Autoimmun 48-49: 31-33, 2014.

10. Giancane G, Consolaro A, Lanni S, Davì S, Schiappapietra B and Ravelli A: Juvenile idiopathic arthritis: Diagnosis and treatment. Rheumatol Ther 3: 187-207, 2016.

11. Clark JD, Flanagan ME and Telliez JB: Discovery and development of Janus kinase (JAK) inhibitors for inflammatory diseases. J Med Chem 57: 5023-5038, 2014.

12. Platanitis E, Demiroz D, Schneller A, Fischer K, Capelle C, Hartl M, Gossenreiter T, Müller M, Novatchkova M and Decker T: A molecular switch from STAT2-IRF9 to ISGF3 underlies interferon-induced gene transcription. Nat Commun 10: 2921, 2019.

13. Schindler C, Levy DE and Decker T: JAK-STAT signaling: From interferons to cytokines. J Biol Chem 282: 20059-20063, 2007.

14. Fragoulis GE, McInnes IB and Siebert S: JAK-inhibitors. New players in the field of immune-mediated diseases, beyond rheumatoid arthritis. Rheumatology (Oxford) 58 (Suppl 1): i43-i54, 2019.

15. Calabresi E, Petrelli F, Bonifacio AF, Puxeddu I and Alunno A: One year in review 2018: Pathogenesis of rheumatoid arthritis. Clin Exp Rheumatol 36: 175-184, 2018.

16. Itoh I, Kasuno K, Yamamoto C, Takahashi N, Shimizu H, Ojima T, Hayashi S, Kimura $\mathrm{H}$ and Iwano M: IgA vasculitis developed as an adverse effect of tofacitinib taken for rheumatoid arthritis. Intern Med 59: 817-821, 2020.

17. Silosi I, Cojocaru M, Foia L, Boldeanu MV, Petrescu F, Surlin P and Biciusca V: Significance of circulating and crevicular matrix metalloproteinase-9 in rheumatoid arthritis-chronic periodontitis association. J Immunol Res 2015: 218060, 2015.

18. Albu CV, Padureanu V, Boldeanu MV, Bumbea AM, Enescu AS, Albulescu DM, Silosi CA and Enescu A: Vascular neurocognitive disorders and the vascular risk factors. J Mind Med Sci 5: 7-15, 2018.

19. Barbulescu AL, Ciurea PL, Mitran C, Chisalau BA, Parvanescu CD, Firulescu SC, Balasoiu M, Boldeanu MV, Popoviciu H and Vreju FA: High frequency ultrasonography of the hand versus anti-RA33 evaluation in early rheumatoid arthritis - a pilot study. Med Ultrason 19: 166-171, 2017.

20. Son HJ, Lee SH, Lee SY, Kim EK, Yang EJ, Kim JK, Seo HB, Park SH, Cho ML and Oncostatin M: Correction: Oncostatin M suppresses activation of IL-17/Th17 via SOCS3 regulation in CD4 ${ }^{+}$T cells. J Immunol 198: 4879, 2017.

21. White UA and Stephens JM: The gp130 receptor cytokine family: Regulators of adipocyte development and function. Curr Pharm Des 17: 340-346, 2011.

22. Lou L, Zhou J, Liu Y, Wei YI, Zhao J, Deng J, Dong B, Zhu L, Wu A, Yang Y, et al: Chlorogenic acid induces apoptosis to inhibit inflammatory proliferation of IL-6-induced fibroblast-like synoviocytes through modulating the activation of JAK/STAT and NF-кB signaling pathways. Exp Ther Med 11: 2054-2060, 2016.

23. Mihara M, Hashizume M, Yoshida H, Suzuki M and Shiina M: IL-6/IL-6 receptor system and its role in physiological and pathological conditions. Clin Sci (Lond) 122: 143-159, 2012.

24. Sheppard M, Laskou F, Stapleton PP, Hadavi S and Dasgupta B: Tocilizumab (Actemra). Hum Vaccin Immunother 13: 1972-1988, 2017.

25. McGovern JL and Notley CA: Production of IL-17: What's STAT got to do with it? JAK-STAT 1: 80-82, 2012. 
26. Siloşi I, Boldeanu MV, Cojocaru M, Biciuşcă V, Pădureanu V, Bogdan M,Badea RG, Avramescu C, Petrescu IO, Petrescu F, et al: The relationship of cytokines IL-13 and IL-17 with autoantibodies profile in early rheumatoid arthritis. J Immunol Res 2016 3109135, 2016.

27. Siloşi I, Boldeanu L, Biciuşcă V, Bogdan M, Avramescu C, Taisescu C, Padureanu V, Boldeanu MV, Dricu A and Siloşi CA: Serum biomarkers for discrimination between hepatitis C-related arthropathy and early rheumatoid arthritis. Int J Mol Sci 18 E1304, 2017.

28. van Hamburg JP and Tas SW: Molecular mechanisms underpinning T helper 17 cell heterogeneity and functions in rheumatoid arthritis. J Autoimmun 87: 69-81, 2018.

29. López de Padilla CM and Niewold TB: The type I interferons: Basic concepts and clinical relevance in immune-mediated inflammatory diseases. Gene 576: 14-21, 2016.

30. Stanifer ML, Pervolaraki K and Boulant S: Differential regulation of type I and type III interferon signaling. Int J Mol Sci 20: $1445,2019$.

31. Zuo J, Xia Y,Li X, Ou-Yang Z and Chen JW: Selective modulation of MAPKs contribute to the anti-proliferative and anti-inflammatory activities of 1,7-dihydroxy-3,4-dimethoxyxanthone in rheumatoid arthritis-derived fibroblast-like synoviocyte MH7A cells. J Ethnopharmacol 168: 248-254, 2015.

32. Malemud CJ: The role of the JAK/STAT signal pathway in rheumatoid arthritis. Ther Adv Musculoskelet Dis 10: 117-127, 2018.

33. Mori M, Hashimoto M, Matsuo T, Fujii T, Furu M, Ito H, Yoshitomi H, Hirose J, Ito Y, Akizuki S, et al: Cell-contactdependent activation of $\mathrm{CD}^{+} \mathrm{T}$ cells by adhesion molecules on synovial fibroblasts. Mod Rheumatol 27: 448-456, 2017.

34. Hammaker D, Nygaard G, Kuhs A, Ai R, Boyle DL, Wang W and Firestein GS: Joint location-specific JAK-STAT signaling in rheumatoid arthritis fibroblast-like synoviocytes. ACR Open Rheumatol 1: 640-648, 2019.

35. O'Shea JJ, Schwartz DM, Villarino AV, Gadina M, McInnes IB and Laurence A: The JAK-STAT pathway: Impact on human disease and therapeutic intervention. Annu Rev Med 66: 311-328, 2015.

36. Busch-Dienstfertig $M$ and González-Rodríguez S: IL-4, JAK-STAT signaling, and pain. JAK-STAT 2: e27638, 2013.

37. Bauer S, Kerr BJ and Patterson PH: The neuropoietic cytokine family in development, plasticity, disease and injury. Nat Rev Neurosci 8: 221-232, 2007.

38. Tan S, Xu J, Lai A, Cui R, Bai R, Li S, Liang W, Zhang G, Jiang S, Liu S, et al: Curculigoside exerts significant anti arthritic effects in vivo and in vitro via regulation of the JAK/STAT/NF $\kappa B$ signaling pathway. Mol Med Rep 19: 2057-2064, 2019.

39. Maeshima K, Yamaoka K, Kubo S, Nakano K, Iwata S, Saito K, Ohishi M, Miyahara H, Tanaka S, Ishii K, et al: The JAK inhibitor tofacitinib regulates synovitis through inhibition of interferon- $\gamma$ and interleukin-17 production by human $\mathrm{CD} 4^{+} \mathrm{T}$ cells. Arthritis Rheum 64: 1790-1798, 2012.

40. Salaffi F, Giacobazzi G and Di Carlo M: Chronic pain in inflammatory arthritis: Mechanisms, metrology, and emerging targets - a focus on the JAK-STAT pathway. Pain Res Manag 2018 8564215,2018

41. Dutzmann J, Daniel JM, Bauersachs J, Hilfiker-Kleiner D and Sedding DG: Emerging translational approaches to target STAT3 signalling and its impact on vascular disease. Cardiovasc Res 106: 365-374, 2015

42. Caruntu C, Boda D, Dumitrascu G, Constantin C and Neagu M: Proteomics focusing on immune markers in psoriatic arthritis. Biomarkers Med 9: 513-528, 2015.

43. Negrei C, Arsene AL, Toderescu CD, Boda D and Ilie M: Acitretin treatment may influence the cell membrane fluidity. Farmacia 60: 767-772, 2012.

44. Negrei C, Ginghină O, Caruntu C, Burcea Dragomiroiu G, Jinescu $G$ and Boda D: Investigation relevance of methotrexate polyglutamates in biological systems by high performance liquid chromatography. Rev Chim-Bucharest 66: 766-768, 2015

45. Negrei C, Caruntu C, Ginghina O, Dragomiroiu GT, Toderescu CD and Boda D: Qualitative and quantitative determination of methotrexate polyglutamates in erythrocytes by high performance liquid chromatography. Rev Chim-Bucharest 66: 607-610, 2015.

46. Boda D, Negrei C, Nicolescu F and Badalau C: Assessment of some oxidative stress parameters in methotrexate treated psoriasis patients. Farmacia 62: 704-710, 2014.

47. Silvagni E, Di Battista M, Bonifacio AF, Zucchi D, Governato G and Scirè CA: One year in review 2019: Novelties in the treatment of rheumatoid arthritis. Clin Exp Rheumatol 37: 519-534, 2019.
48. Choy EH: Clinical significance of Janus kinase inhibitor selectivity. Rheumatology (Oxford) 58: 953-962, 2019.

49. Hodge JA, Kawabata TT, Krishnaswami S, Clark JD, Telliez JB, Dowty ME, Menon S, Lamba M and Zwillich S: The mechanism of action of tofacitinib - an oral Janus kinase inhibitor for the treatment of rheumatoid arthritis. Clin Exp Rheumatol 34: 318-328, 2016.

50. Rigby WFC, Lampl K, Low JM and Furst DE: Review of routine laboratory monitoring for patients with rheumatoid arthritis receiving biologic or nonbiologic DMARDs. Int J Rheumatol 2017: 9614241, 2017.

51. Banerjee S, Biehl A, Gadina M, Hasni S and Schwartz DM: JAK-STAT signaling as a target for inflammatory and autoimmune diseases: Current and future prospects. Drugs 77: 521-546, 2017.

52. Jegatheeswaran J, Turk M and Pope JE: Comparison of Janus kinase inhibitors in the treatment of rheumatoid arthritis: A systemic literature review. Immunotherapy 11: 737-754, 2019.

53. Taylor PC: Clinical efficacy of launched JAK inhibitors in rheumatoid arthritis. Rheumatology (Oxford) 58 (Suppl 1): i17-i26, 2019

54. Borie DC, Larson ML, Flores MG, Campbell A, Rousvoal G, Zhang S, Higgins JP, Ball DJ, Kudlacz EM, Brissette WH, et al: Combined use of the JAK3 inhibitor CP-690,550 with mycophenolate mofetil to prevent kidney allograft rejection in nonhuman primates. Transplantation 80: 1756-1764, 2005

55. Zerbini CA and Lomonte AB: Tofacitinib for the treatment of rheumatoid arthritis. Expert Rev Clin Immunol 8: 319-331, 2012.

56. Kremer JM, Bloom BJ, Breedveld FC, Coombs JH, Fletcher MP, Gruben D, Krishnaswami S, Burgos-Vargas R, Wilkinson B, Zerbini CA, et al; Tofacitinib Study Investigators: The safety and efficacy of a JAK inhibitor in patients with active rheumatoid arthritis: Results of a double-blind, placebo-controlled phase IIa trial of three dosage levels of CP-690,550 versus placebo. Arthritis Rheum 60: 1895-1905, 2009.

57. Tanaka Y, Suzuki M, Nakamura H, Toyoizumi S and Zwillich SH; Tofacitinib Study Investigators: Phase II study of tofacitinib (CP-690,550) combined with methotrexate in patients with rheumatoid arthritis and an inadequate response to methotrexate. Arthritis Care Res (Hoboken) 63: 1150-1158, 2011.

58. Fleischmann R, Cutolo M, Genovese MC, Lee EB, Kanik KS, Sadis S, Connell CA, Gruben D, Krishnaswami S, Wallenstein G, et al: Phase IIb dose-ranging study of the oral JAK inhibitor tofacitinib (CP-690,550) or adalimumab monotherapy versus placebo in patients with active rheumatoid arthritis with an inadequate response to disease-modifying antirheumatic drugs. Arthritis Rheum 64: 617-629, 2012.

59. Mogul A, Corsi K and McAuliffe L: Baricitinib: The second FDA-approved JAK inhibitor for the treatment of rheumatoid arthritis. Ann Pharmacother 53: 947-953, 2019.

60. Kubo S, Nakayamada S and Tanaka Y: Baricitinib for the treatment of rheumatoid arthritis. Expert Rev Clin Immunol 12: 911-919, 2016.

61. Al-Salama ZT and Scott LJ: Baricitinib: A review in rheumatoid arthritis. Drugs 78: 761-772, 2018.

62. Biggioggero M, Becciolini A, Crotti C, Agape E and Favalli EG: Upadacitinib and filgotinib: The role of JAK1 selective inhibition in the treatment of rheumatoid arthritis. Drugs Context 8: 212595, 2019.

63. Takeuchi T, Tanaka Y, Iwasaki M, Ishikura H, Saeki S and Kaneko Y: Efficacy and safety of the oral Janus kinase inhibitor peficitinib (ASP015K) monotherapy in patients with moderate to severe rheumatoid arthritis in Japan: A 12-week, randomised, double-blind, placebo-controlled phase IIb study. Ann Rheum Dis 75: 1057-1064, 2016.

64. Quintás-Cardama A, Kantarjian H, Cortes J and Verstovsek S Janus kinase inhibitors for the treatment of myeloproliferative neoplasias and beyond. Nat Rev Drug Discov 10: 127-140, 2011.

65. MacFarlane LA and Todd DJ: Kinase inhibitors: The next generation of therapies in the treatment of rheumatoid arthritis. Int J Rheum Dis 17: 359-368, 2014.

66. Gadina M, Schwartz DM and O'Shea JJ: Decernotinib: A next-generation Jakinib. Arthritis Rheumatol 68: 31-34, 2016.

67. Genovese MC, van Vollenhoven RF, Pacheco-Tena C, Zhang Y and Kinnman N: VX-509 (decernotinib), an oral selective JAK-3 inhibitor, in combination with methotrexate in patients with rheumatoid arthritis. Arthritis Rheumatol 68: 46-55, 2016.

This work is licensed under a Creative Commons Attribution-NonCommercial-NoDerivatives 4.0 International (CC BY-NC-ND 4.0) License. 